\title{
Produção de leite de vacas da raça Holandesa de pequeno, médio e grande porte
}

\author{
Milk production of Holstein cows in small, medium and large size
}

\author{
Décio Adair Rebellatto da Silva ${ }^{\mathrm{I}}$ Clair Jorge Olivo ${ }^{\mathrm{II}}$ Ben-Hur Costa de Campos ${ }^{\mathrm{II}}$ \\ Thiago Moreira Tejkowski ${ }^{\text {IV }}$ Gilmar Roberto Meinerz ${ }^{\text {II }}$ Ana Gabriela de Freitas Saccol ${ }^{\text {V }}$ \\ Silvio Teixeira da $\operatorname{Costa}^{\mathrm{v}}$
}

RESUMO

O objetivo deste estudo foi avaliar o efeito do composto corporal sobre a produção de leite de vacas da raça Holandesa de primeira, segunda e terceira lactação. Para avaliação, foram usadas 133 vacas, selecionadas de um rebanho de 280 animais em lactação, classificadas como pequenas, médias e grandes, com base no composto corporal (estatura $\times 10+$ força $\times 5+$ profundidade $\times 3+$ largura de garupa $\times 2) / 20$. Os dados foram coletados, entre janeiro de 2007 e outubro de 2009, de um rebanho comercial com sistema intensivo de produção de leite, localizado em Salto do Jacuí, Rio Grande do Sul, Brasil. Todas as vacas foram submetidas às mesmas condições de instalações e ordenhadas três vezes ao dia. Os dados foram obtidos com auxílio do software AFIMILK ${ }^{\circledast}$ - SAE AFIKIM. O delineamento experimental utilizado foi o inteiramente casualizado com três tratamentos (tamanho corporal). Não foi verificada interação entre a produção de leite e composto corporal. Foi observada baixa correlação entre composto corporal e produção de leite. Vacas de porte médio e grande apresentaram produção de leite similar e superior $(P<0,05)$ às pequenas. As médias de produção de leite para vacas pequenas, médias e grandes em 305 dias foram de 8.053; 8.810 e 9.218 litros, respectivamente. Com base na totalidade das lactações avaliadas, as vacas de porte médio são mais eficientes que as demais, considerando o sistema intensivo de produção em análise.

Palavras-chave: composto corporal, dimensões corporais, persistência, peso corporal, pico de produção de leite.

\begin{abstract}
The objective of this study was to evaluate the effect of the body size in milk production in the first, second and third lactations of Holstein cows. A total 133 cows were used, selected from a herd of 280 cows, classified as small, medium and large based on the body size composite (stature $x 10+$ strength $\times 5+$ depth $\times 3+$ rump width $\times 2$ )/20. Data were collected from January 2007 to October 2009 in a commercial herd with intensive system of milk production, located in Salto Jacuí, Rio Grande do Sul, Brazil. All cows were subjected to the same conditions of facilities management being used and milked three times a day. Data were obtained from the software Afimilk ${ }^{\circledR}$ - SAE AFIKIM. The experimental design was a completely randomized design with three treatments (body size). There was no interaction between milk production and body composite. Low correlation among body size composite and milk production was observed. Cows for medium and large size had similar on milk production superior $(P<0,05)$ to small size. Means for 305-days milk production in the lactation were 8,053; 8,810 and 9,218 liters for small, medium and large cows, respectively. Based on the totality of lactations evaluated, cows of medium size were more efficient than the others for the intensive production system in question.
\end{abstract}

Key words: body size, body size composite, body weight, peak milk production, persistency.

\section{INTRODUÇÃO}

A atividade leiteira está presente em cerca de 1,3 milhões de propriedades brasileiras, sendo

'Curso de Medicina Veterinária, Universidade Federal do Pampa (UNIPAMPA), CP 118, BR 472, km 592, 97500-970, Uruguaiana, RS, Brasil. E-mail: rebellattodecio@ig.com.br. Autor para correspondência.

"Departamento de Zootecnia, Centro de Ciências Rurais (CCR), Universidade Federal de Santa Maria (UFSM), Santa Maria, RS, Brasil.

IIIInstituto Federal do Rio Grande do Sul (IFRS), Ibirubá, RS, Brasil.

${ }^{\text {Iv }}$ Departamento de Ciências da Saúde, Curso de Medicina Veterinária, Fundação Universidade de Cruz Alta (UNICRUZ), Cruz Alta, RS, Brasil.

${ }^{v}$ Centro de Educação Superior Norte (CESNORS), Palmeira das Missões, RS, Brasil. 
considerada uma das mais importantes da agropecuária (IBGE, 2008). Para ter eficiência nesta atividade, vários fatores devem ser considerados, destacando-se a raça e o tipo animal. No contexto atual, o fator mais importante de seleção é a produção de leite. Para tanto, utilizam-se metodologias que auxiliam no melhoramento genético dos animais, nem sempre associado às condições ambientais que favoreçam a expressão das características desejadas (ROUGOOR et al., 1997).

O melhoramento genético é uma ferramenta importante para a cadeia produtiva do leite, havendo muitos estudos nos quais são correlacionados fatores envolvidos diretamente na produção e fatores nãoprodutivos, facilmente mensuráveis. Dentre estes, destacam-se o peso corporal, altura e largura torácica (LAMB et al., 1977). Em pesquisa conduzida com vacas das raças Parda Suíço, Ayshire e Holandesa, foram observadas correlações negativas entre essas medidas e a eficiência alimentar (DICKINSON et al., 1969). Esta pesquisa resultou da hipótese de que vacas menores seriam mais eficientes que vacas maiores. Nesse contexto, o tamanho de vaca tem recebido grande ênfase em programas de seleção, havendo poucas evidências de que vacas maiores são mais vantajosas (MAHONEY et al., 1986). Alguns pesquisadores demonstraram que vacas da raça Holandesa de porte menor têm uma eficiência alimentar maior do que as vacas grandes (YEREX et al., 1988). Também se demonstrou que vacas da raça Holandesa de menor porte produzem mais leite por unidade de peso corporal e por unidade de alimento consumido do que vacas de maior porte (DONKER et al., 1983).

Contrariando esses estudos, o que se tem observado, na América do Norte, especialmente, são animais cada vez maiores, como resultado da seleção genética, visando ao aumento da produção de leite individual. No Brasil, isso se deve, principalmente, ao uso da inseminação artificial de vacas com sêmen de touros provenientes daquela região. Em um estudo com vacas da raça Holandesa de pequeno e grande porte, submetidas às mesmas condições ambientais, observou-se que não há diferenças significativas nas produções de leite, proteína e gordura entre esses grupos, confirmando a maior eficiência de vacas de menor porte (CARVALHO, 2000).

Nos últimos anos, a Associação Americana de Criadores da raça Holandesa alterou os parâmetros de classificação dos animais para uma forma descrita como composto corporal. A antiga classificação enfatizava traços de estatura, massa corporal, profundidade, ângulo e largura da garupa, com um peso semelhante para cada um. Para o novo cálculo, a proporção é de 10: 5: 3: 2 para a estatura, força, profundidade e largura de garupa. Em contrapartida, o novo composto corporal apresenta mais informações sobre o tamanho da vaca, anteriormente baseado na estatura. A nova classificação proporciona aos produtores informações mais precisas das características corporais desejáveis a serem transmitidas às futuras gerações (PLOURD, 2000). Em vários estudos experimentais, fez-se o uso de medidas corporais, associando com tamanho e peso de vacas leiteiras (YEREX et al., 1988; HEINRICHS et al., 1992; ENEVOLDSEN \& KRISTENSEN, 1997; HANSEN et al., 1999a; DINGWELL et al., 2006).

Em países como os EUA, onde os subsídios agrícolas são elevados, o custo operacional de vacas de grande porte não é muito considerado. $\mathrm{O}$ aumento na produção é o objetivo principal dos produtores americanos, sendo também uma realidade em países da comunidade européia e parte da América Latina. Ao se selecionar os animais com maior produção, elevando o peso, consequentemente, tem-se observado uma constante redução na fertilidade desses rebanhos, constatando-se uma correlação negativa entre fertilidade e produção de leite (MADALENA, 2008). Além disso, esses animais são mais suscetíveis a outros problemas de saúde (WINDIG et al., 2006).

Nos EUA, foi relatado um aumento na incidência de casos de mastite $(20 \%)$ e claudicação $(10 \%)$, dentre outras intervenções de saúde, em uma avaliação realizada de janeiro de 2001 a dezembro de 2003 (ZWALD et al., 2004). Associado a esta deterioração da saúde dos animais de alta produção, há a diminuição na sua vida útil, interferindo diretamente nos ganhos com a produção. No Brasil, em virtude da importação de material genético de animais de alta produção, esses problemas começam a afetar os rebanhos, sendo crescente a preocupação com a queda da fertilidade e com o aumento de problemas de úbere, cascos, metabólicos e na reprodução nos rebanhos leiteiros (MADALENA, 2008).

Nesse contexto, a presente pesquisa tem como objetivo comparar os diferentes grupos de vacas da raça Holandesa, de acordo com o composto corporal, com relação à produção de leite, em um rebanho de um sistema intensivo de produção da Mesorregião do Noroeste do Rio Grande do Sul.

\section{MATERIAL E MÉTODOS}

A pesquisa foi realizada na fazenda Salto do Jacuí Grande, localizada no município de Salto do JacuíRS, Mesorregião Noroeste do RS, com clima subtropical úmido, latitude $-29^{\circ} 05^{\circ} 18^{\prime \prime}$ e longitude $53^{\circ} 12^{\circ} 45^{\prime \prime}$, precipitação média mensal de $116,26 \mathrm{~mm}$, média anual de $18^{\circ} \mathrm{C}$, altitude de $322 \mathrm{~m}$ e predominância de latossolo.

Foram utilizadas 133 vacas da raça Holandesa representativas do rebanho, com média de 266 dias de lactação, as quais foram submetidas à 
biometria em janeiro de 2008. Esses animais, já identificados, foram previamente pesados, avaliados quanto ao escore de condição corporal e classificados de acordo com o composto corporal, utilizado pela Associação Americana de Criadores da Raça Holandesa para classificação em tamanho. Para tanto, fez-se a medição da altura compreendida entre a distância vertical da proeminência do íleo e o talão do casco posterior; da força, estabelecida pela distância horizontal entre as partes ventrais dos úmeros; profundidade, entre a linha lombar e ventral, na altura da última costela; e largura da garupa, medida entre as laterais opostas dos ísquios. Essas quatro medidas corporais foram utilizadas no cálculo do composto de tamanho corporal individual, obtido pela seguinte fórmula: (estatura $\times 10+$ força $\times 5+$ profundidade $\times 3+$ largura de garupa $\times 2$ ) $20^{-1}$ (PLOURD, 2000). Os valores envolvendo os grupos de vacas experimentais pequenas e grandes foram obtidos a partir de $1 / 3$ nos pontos extremos, em direção à média do composto corporal; os valores intermediários restantes constituíram-se no grupo de vacas médias. Assim, o grupo das pequenas e o das grandes foi formado por 44 animais cada, enquanto o grupo das médias por 45 animais. As classes de composto corporal para cada grupo variaram de 89,6 a 95,2 pontos para as vacas pequenas; de 95,3 a 97,9 para vacas médias e de 98,0 a 104,5 pontos para vacas grandes (Tabela 1). Para avaliação, foram considerados animais da primeira, segunda e terceira lactações, coletando-se dados da produção diária e total aos 305 dias.

As vacas foram submetidas diariamente a três ordenhas com intervalos regulares de oito horas, às $3 \mathrm{~h}, 11 \mathrm{~h}$ e $19 \mathrm{~h}$. Cada animal recebia diariamente complementação alimentar com $20 \mathrm{~kg}$ de silagem de milho e $12 ; 10,5 ;$ e $7,5 \mathrm{~kg}$ de concentrado (com $20 \%$ de proteína bruta), para os lotes que produzem 37 ou mais,
22 a 36 e 12 a 21 litros de leite vaca $^{-1}$ dia $^{-1}$, respectivamente. Após essa alimentação, as vacas eram conduzidas para áreas de pastagens constituídas por aveia (Avena strigosa) e azevém (Lolium multiflorum), entre maio e outubro, e com Tifton 85 (Cynodon spp.) e sorgo forrageiro (Sorghum spp.), entre novembro e abril. A partir dos 60 dias de lactação, as vacas receberam aplicações intramusculares de BST (somatrotopina bovina), a cada 14 dias. O tratamento foi interrompido 30 dias antes da secagem. O controle da produção foi realizado através do sistema de coleta de dados AFIMILK ${ }^{\circledR}$ - SAE AFIKIM em cada ordenha.

$\mathrm{O}$ delineamento experimental foi o inteiramente casualizado, com três tratamentos (tamanhos de vacas) e número variável de repetições (vacas), em três lactações. Os dados foram ajustados pelo teste de Cook para retirada de outliers e, posteriormente, submetidos à análise de variância. Quando detectadas diferenças, as médias foram comparadas entre si pelo teste de Duncan em nível de $5 \%$ de probabilidade do erro. Também foram realizadas análises de correlação entre as variáveis da curva de lactação com as medidas corporais e de regressão, envolvendo períodos da lactação. Para as análises, foi utilizado o pacote estatístico SAS, versão 6.08 (SAS, 1997).

O modelo estatístico referente à análise das variáveis estudadas foi representado por:

$Y_{i j k}=\mu+T_{i}+R_{j}\left(T_{i}\right)+L_{k} x T_{i}+L_{x t}+\varepsilon_{i j k}$, em que $Y_{i j k}$ representa as variáveis dependentes; $\mu$ é a média de todas as observações; $\mathrm{T}_{\mathrm{i}}$ é o efeito de tratamento (composto corporal); $\mathrm{R}_{\mathrm{j}}\left(\mathrm{T}_{\mathrm{i}}\right)$ é o efeito das repetições nos tratamentos (erro a); $\mathrm{L}_{k}$ é o efeito da ordem de lactação; $L_{k} x T_{i}$ representa a interação entre tratamentos e lactações; e $\varepsilon_{\mathrm{ijk}}$ corresponde ao erro experimental residual (erro b).

Tabela 1 - Valores médios do composto corporal, dos componentes do composto corporal e de variáveis complementares de vacas da raça Holandesa. Salto do Jacuí, RS, 2008.

\begin{tabular}{|c|c|c|c|c|}
\hline \multirow{2}{*}{ Variáveis } & \multicolumn{3}{|c|}{----------------------------------------Vacas------------------------------------ } & \multirow{2}{*}{ Média } \\
\hline & Pequenas & Médias & Grandes & \\
\hline Composto corporal & $93,5 \pm 0,71$ & $97,0 \pm 0,82$ & $100,0 \pm 0,78$ & $96,83 \pm 3,25$ \\
\hline Estatura $(\mathrm{cm})$ & $132,77 \pm 1,93$ & $137,87 \pm 1,94$ & $143,72 \pm 2,72$ & $138,12 \pm 5,48$ \\
\hline Força $(\mathrm{cm})$ & $46,05 \pm 2,54$ & $45,63 \pm 2,43$ & $49,03 \pm 2,67$ & $46,9 \pm 1,85$ \\
\hline Profundidade $(\mathrm{cm})$ & $80,40 \pm 2,41$ & $83,58 \pm 2,14$ & $86,05 \pm 2,88$ & $83,34 \pm 2,83$ \\
\hline Largura da garupa $(\mathrm{cm})$ & $36,82 \pm 2,10$ & $37,77 \pm 2,48$ & $39,58 \pm 2,84$ & $38,06 \pm 1,4$ \\
\hline Peso $(\mathrm{kg})$ & $555,89 \pm 53,49$ & $583,38 \pm 41,44$ & $636,15 \pm 53,54$ & $591,81 \pm 40,79$ \\
\hline Escore corporal & $3,75 \pm 0,29$ & $3,57 \pm 0,39$ & $3,7 \pm 0,36$ & $3,67 \pm 0,09$ \\
\hline Dias em lactação $^{1}$ & $263,23 \pm 139,82$ & $261,67 \pm 129,76$ & $272,70 \pm 156,50$ & $265,87 \pm 141,36$ \\
\hline
\end{tabular}

Valores expressos em média e ( \pm ) desvio padrão; Vacas pequenas, médias e grandes, n 133, com composto corporal entre 89,6 e 95,2; 95,3 e 97,$9 ; 98,0$ e 104,5, respectivamente. Valores expressos como média aritmética e ( \pm ) desvio padrão; ${ }^{1}$ Dia em que o animal foi medido. 


\section{RESULTADOS E DISCUSSÃO}

Analisando-se os diferentes grupos constituídos (Tabela 1), observa-se que há similaridade entre eles em variáveis fundamentais de comparação. Os valores estimados de escore corporal, com padrão superior a 3,5, sugerem que os animais receberam manejo condizente com suas necessidades nutricionais. O escore corporal médio dos animais de 3,67 está próximo do padrão recomendado para o final da lactação (LAGO et al., 2001), indicando que as vacas tiveram condições de expressar seu potencial de produção de leite. Também o período de lactação, semelhante entre os lotes, contribuiu para uma comparação mais efetiva, à medida que os animais estavam submetidos a condições ambientais e de manejo similares, como pode ser constatado pelo dia de coleta de dados.

Comparativamente, observam-se que o grupo de animais, representativo da propriedade, apresenta uma diversidade de tamanhos que já não é observada em rebanhos de países como os EUA, de onde provém boa parte do material genético de animais da raça Holandesa. Estudos conduzidos em épocas distintas demonstram que houve aumento gradativo do peso e do tamanho dos animais da raça Holandesa criados nos EUA (MAHONEY et al., 1986). Em pesquisas conduzidas por YEREX et al. (1988), com dados de rebanhos criados entre as décadas de 60 e 80 , verificou-se que as vacas, após o parto, possuíam entre 555 e $609 \mathrm{~kg}$. Mais tarde, HANSEN et al. (1999) relataram que vacas, na mesma condição fisiológica, apresentaram peso entre 641 e $720 \mathrm{~kg}$. Também nos EUA, KERTZ et al. (1997) verificaram peso médio de $718,38 \mathrm{~kg}$ para vacas da raça Holandesa na terceira lactação, confirmando o aumento que vem ocorrendo no tamanho dos animais da raça Holandesa nos rebanhos americanos.

Os resultados da análise de variância demonstraram que não houve interação entre o tamanho do animal e as lactações (Tabela 2). Para a produção diária de leite, houve diferença $(\mathrm{P}<0,05)$, com menor valor de produção, dos animais de pequeno porte, havendo comportamento similar entre vacas de médio e grande porte. Para as lactações, houve diferença $(\mathrm{P}<0,05)$ com menor produção para os animais de primeira cria. Esse resultado pode ser atribuído, em parte, à condição das vacas primíparas, que ainda estão crescendo, sendo mais uma variável que interfere na produção, implicando maior variabilidade dos dados (MATOS et al., 1997; COFFEY et al., 2006) em relação às demais lactações. Na segunda e terceira lactações, período em que os animais estão próximos da idade adulta, fase em que os animais tendem a expressar seu potencial máximo de produção (MATOS et al., 1997) e a interferência do crescimento é pequena, os valores são semelhantes, não havendo interferência do tamanho. Comportamento similar ao da produção ao pico foi observado com a produção total, em 305 dias de lactação, não havendo diferença entre as vacas médias e grandes e entre a segunda e terceira lactações. Comparativamente, os valores obtidos da produção de leite em 305 dias na primeira lactação são similares aos verificados no Canadá, com $7.717 \mathrm{~kg}$ (CHRISTENSEN et al., 1993), e maiores em relação aos observados em animais registrados da raça Holandesa no Rio Grande Sul (MATOS et al., 1997). Produções menores foram obtidas em levantamentos feitos no estado do Paraná, com dados de 75 mil animais,

Tabela 2 - Média de produção de leite diária e em 305 dias de lactação de vacas da raça Holandesa de tamanhos diferentes. Salto do Jacuí, RS, 2008.

\begin{tabular}{|c|c|c|c|c|}
\hline \multirow{3}{*}{ Vacas } & \multicolumn{3}{|c|}{ 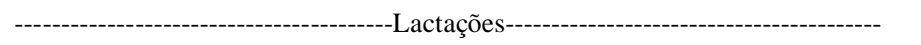 } & \multirow{3}{*}{ Média } \\
\hline & & & & \\
\hline & $1^{\mathrm{a}}$ & $2^{-\underline{a}}$ & $3^{\mathrm{a}}$ & \\
\hline & \multicolumn{4}{|c|}{ - } \\
\hline Pequenas & $20,99 \pm 1,84$ & $28,38 \pm 2,50$ & $30,08 \pm 4,58$ & $25,44 \pm 3,92^{\mathrm{B}}$ \\
\hline Médias & $23,55 \pm 3,77$ & $31,36 \pm 3,01$ & $31,86 \pm 2,17$ & $28,92 \pm 5,03^{\mathrm{A}}$ \\
\hline Grandes & $24,58 \pm 5,04$ & $31,95 \pm 4,27$ & $32,64 \pm 2,37$ & $29,72 \pm 4,94^{\mathrm{A}}$ \\
\hline \multirow[t]{2}{*}{ Média } & $23,04 \pm 1,85^{\mathrm{b}}$ & $30,56 \pm 2,32^{\mathrm{a}}$ & $31,07 \pm 3,58^{\mathrm{a}}$ & \\
\hline & \multicolumn{4}{|c|}{ 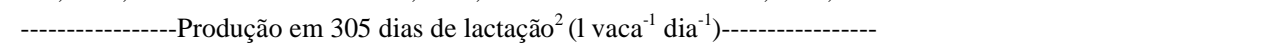 } \\
\hline Pequenas & $6.339,00 \pm 542,19$ & $8.828,00 \pm 406,64$ & $9.173,00 \pm 1.423,00$ & $8.113,00 \pm 1.486,81^{\mathrm{B}}$ \\
\hline Médias & $7.285,36 \pm 1.157,72$ & $9.671,00 \pm 699,52$ & $9.719,00 \pm 962,81$ & $8.892,00 \pm 1.323,73^{\mathrm{A}}$ \\
\hline Grandes & $7.498,00 \pm 1.537,41$ & $9.745,00 \pm 1.191,00$ & $9.954,00 \pm 961,82$ & $9.066,00 \pm 1490,72^{\mathrm{A}}$ \\
\hline Média & $7.040,79 \pm 616,00^{\mathrm{b}}$ & $9.415,33 \pm 664,83^{\mathrm{a}}$ & $9.615,00 \pm 511,79^{\mathrm{a}}$ & \\
\hline
\end{tabular}

1,2 Dados de 125 lactações. Vacas pequenas, médias e grandes com composto corporal entre 89,6 e 95,2; 95,3 e 97,9; 98,0 e 104,5, respectivamente. Valores expressos como média aritmética e $( \pm)$ desvio padrão; Médias seguidas por letras distintas na coluna diferem entre si pelo teste de Duncan a 5\% de probabilidade do erro. 
revelando uma produção média de $6.930 \mathrm{~kg}$ (MOLENTO et al., 2004). Esses resultados apontam que as vacas de porte médio são mais adequadas à propriedade, por não diferirem das grandes e serem, consequentemente, mais eficientes na produção de leite, à medida que demonstram menor gasto energético com a manutenção (HANSEN, 2000). Houve diferença demonstrada nos dados médios de produção entre os três grupos de vacas, sendo favorável a vacas médias e grandes. No entanto, vacas pequenas, na terceira lactação, apresentam variação acentuada de produção individual, sugerindo que nesse rebanho em análise existem vacas pequenas que se sobressaem com altas produções, porém o grupo produziu menos que os demais. As vacas de menor tamanho podem ser adequadas ao sistema, mesmo produzindo menos que as grandes, essa assertiva tem como base a pesquisa experimental, que demonstrou que as vacas da raça Holandesa de menor tamanho são mais eficientes no aproveitamento de alimentos (DONKER et al., 1983), (DICKINSON et al., 1969; LAMB et al., 1977; YEREX et al., 1988), além de apresentarem maior vida útil e menos problemas sanitários.

Dessa forma, a estratégia de elevar a produção de leite e o tamanho dos animais é inadequada, considerando sua associação com problemas reprodutivos e problemas de saúde (MAHONEY et al., 1986: ZWALD et al., 2004), com destaque para mastite (HERINGSTAD et al., 2003). Esses problemas têm crescido também no rebanho brasileiro (MADALENA, 2008), especialmente em vacas da raça Holandesa (WOLFF et al., 2004).

Os resultados da análise de correlação, usando-se dados de todos os animais, confirmam a baixa associação entre a produção de leite e o composto corporal $(0,32 ; \mathrm{P}=0,01)$ e com os componentes do composto, estatura $(0,295 ; \mathrm{P}=0,0188)$ e profundidade $(0,288 ; \mathrm{P}=0,022)$; não foram observadas correlações com a força e a largura da garupa, como verificada $(0,37)$ por ESTEVES et al.(2004). Esses resultados indicam que o aumento na produção de leite é influenciado de forma branda pelas características que conferem maior tamanho aos animais.

Essa assertiva pode ser confirmada pela pesquisa conduzida por HANSEN et al. (1999a), segundo a qual vacas de linhagens com tamanhos distintos apresentam diferentes variações em suas características físicas, mas semelhanças na produção de leite, sendo um indício de que as vacas menores são mais eficientes. Orientação similar tem sido apontada, indicando que a diminuição do tamanho seria um fator importante para os sistemas de produção de leite do Brasil (MARTINS et al., 2003).

\section{CONCLUSÃO}

As vacas de tamanho médio da raça Holandesa apresentam desempenho similar às vacas grandes na produção de leite, sendo assim as mais indicadas no sistema de produção analisado. Existe a necessidade de maior mensuração de dados de vacas pequenas, as quais podem ser interessantes nos sistemas de produção. Há necessidade de outros trabalhos, com avaliação de vacas holandesas de diferentes tamanhos, contemplando produção aliada ao consumo, conversão alimentar e longevidade.

\section{REFERÊNCIAS}

CARVALHO, M.P. Qual o tamanho ideal das vacas leiteiras? Milkpoint, 2000. Disponível em: <http://www.milkpoint.com.br/ qual-o-tamanho-ideal-das-vacas-leiteiras $>$. Acesso em: 25 mar. 2008 .

COFFEY, M. P. et al. Genetic aspects of growth of HolsteinFriesian dairy cows from birth to maturity. Journal of Dairy Science, v.89, n.1, p.322-329, 2006. Disponível em: <http:/ / w w w.journalofdairy s c i ence.org/article/ PIIS0022030206720975/fulltext>. Acesso em: 15 dez. 2010. doi:10.3168/jds.S0022-0302(06)72097-5.

CHRISTENSEN, D.A.; FEHR, M.I. The dairy industry. In: MARTIN, J.R. et al. Animal production in Canadá. Edmonton, Alberta: Faculty of Extension, University of Alberta, 1993. p.7592.

DICKINSON, F.N. et al. Comparative efficiency of feed utilization during first lactation of Ayrshire, Brown Swiss, and Holstein cows. Journal of Dairy Science, v.52, n.4, p.489-497, 1969. Disponível em: <http://download.journals.elsevierhealth.com/pdfs/ journals/0022-0302/PIIS0022030269865938.pdf>. Acesso em: 15 dez. 2010. doi:10.3168/jds.S0022-0302(69)86593-8.

DINGWELL, R.T. et al. An evaluation of two indirect methods of estimating body weight in Holstein calves and heifers. Journal of Dairy Science, v.89, n.10, p.3992-3998, 2006. Disponível em: <http://www.journalofdairyscience.org/article/ PIIS0022030206724420/fulltext>. Acesso em: 15 dez. 2010. doi: 10.3168/jds.S0022-0302(06)72442-0.

DONKER, J.D. et al. Feed intake and milk production from tree rates of concentrate for cows bred to differ in size. Journal of Dairy Science, v.66, n.6, p.1337-1348, 1983. Disponível em: <http://download.journals.elsevierhealth.com/pdfs/journals/ 0022-0302/PIIS0022030283819432.pdf>. Acesso em: $15 \mathrm{dez}$. 2010. doi: 10.3168/jds.S0022-0302(83)81943-2.

ENEVOLDSEN, C.; KRISTENSEN, T. Estimation of body weight from body size measurements and body condition scores in dairy cows. Journal of Dairy Science, v.80, n.9, p.1988-1995, 1997. Disponível em: <http://download.journals.elsevierhealth.com/pdfs/ journals/0022-0302/PIIS0022030297761423.pdf>. Acesso em: 15 dez. 2010. doi:10.3168/jds.S0022-0302(97)76142-3.

ESTEVES, A.M.C. et al. Correlações genéticas e fenotípicas entre características de tipo e produção de leite em bovinos da raça holandesa. Arquivo Brasileiro de Medicina Veterinária e Zootecnia, v.56, n.4, p.529-535, 2004. Disponível em: <http://www.scielo.br/pdf/rbz/v32n6/ 18431.pdf>. Acesso em: 15 dez. 2010. doi: 10.1590/S010209352004000400015 . 
HANSEN, L.B. et al. Longevity of Holstein cows bred to be large versus small for body size. Advances in Dairy Technology, v.11, n.1, p. 39, 1999a.

HANSEN, L.B. et al. Productive life and reasons for disposal of holstein cows selected for large versus small body size. Journal of Dairy Science, v.82, n.4, p.795-801, 1999b. Disponível em: <http://download.journals.elsevierhealth.com/ pdfs/journals/0022-0302/PIIS0022030299752987.pdf>. Acesso em: 15 dez. 2010. doi:10.3168/jds.S00220302(99)75298-7.

HANSEN, L.B. Consequences of Selection for milk yield from a geneticist's viewpoint. Journal of Dairy Science, v.83, n.5, p.1145-1150, 2000. Disponível em: <http:// download.journals.elsevierhealth.com/pdfs/journals/00220302/PIIS0022030200749800.pdf>. Acesso em: $15 \mathrm{dez}$. 2010. doi:10.3168/jds.S0022-0302(00)74980-0.

HEINRICHS, A.J. et al. Predicting body weight and wither height in Holstein heifers using body measurements. Journal of Dairy Science, v.75, n.12, p.3576-3581, 1992. Disponível em: <http://download.journals.elsevierhealth.com/pdfs/journals/ 0022-0302/PIIS002203029278134X.pdf>. Acesso em: $15 \mathrm{dez}$. 2010. doi:10.3168/jds.S0022-0302(92)78134-X.

HERINGSTAD, B. et al. Selection responses for clinical mastitis and protein yield in two norwegian dairy cattle selection experiments. Journal of Dairy Science, v.86, n.9, p.2990-2999, 2003. Disponível em: <http://www.journalofdairyscience.org/article/ PIIS0022030203738971/fulltext>. Acesso em: 15 dez. 2010. doi:10.3168/jds.S0022-0302(03)73897-1.

IBGE. Instituto Brasileiro de Geografia e Estatística. Disponível em: <http://www.ibge.gov.br/>. Acesso em: 08 jan 2008.

KERTZ. A.F. et al. Body weight, body condition score, and wither height of prepartum Holstein cows and birth weight and sex of calves by parity: a database and summary. Journal of Dairy Science, v.80, n.3, p.525-529, 1997. Disponível em: <http://download.journals.elsevierhealth.com/pdfs/journals/ 0022-0302/PIIS0022030297759666.pdf>. Acesso em: $15 \mathrm{dez}$. 2010. doi:10.3168/jds.S0022-0302(97)75966-6.

LAGO, E.P. et al. Efeito da condição corporal ao parto sobre alguns parâmetros do metabolismo energético produção de leite e incidência de doenças no pós-parto de vacas leiteiras. Revista Brasileira de Zootecnia, v.30, n.5, p.1544-1549, 2001. Disponível em: <http://www.scielo.br/pdf/rbz/v37n1/ v37n1a17.pdf>. Acesso em: 15 dez. 2010. doi: 10.1590/ S1516-35982008000100017.

LAMB, R.C. et al. Effects of sire and interaction of sire with ration on efficiency of feed utilization by Holsteins. Journal of Dairy Science, v. 60, n.10, p.1755-1767, 1977. Disponível em: <http://download.journals.elsevierhealth.com/pdfs/journals/ 0022-0302/PIIS002203027784099X.pdf>. Acesso em: 15 dez. 2010. doi:10.3168/jds.S0022-0302(77)84099-X.

MADALENA, F.H. Efeitos colaterais da genética de alta produção. Milkpoint, 2008. Disponível em: <www.milkpoint.com.br/efeitoscolaterais-da-genética-de-alta-produção〉. Acesso em: 23 maio, 2008.

MAHONEY, C.B. et al. Health care of Holsteins selected for large or small body size. Journal of Dairy Science, v.69, n.12, p.3131-3139, 1986. Disponível em: <http:// download.journals.elsevierhealth.com/pdfs/journals/0022-
0302/PIIS0022030286807779.pdf>. Acesso em: 15 dez. 2010. doi:10.3168/jds.S0022-0302(86)80777-9.

MARTINS, G.A. et al. Objetivos econômicos de seleção de bovinos de leite para fazenda demonstrativa na Zona da Mata de Minas Gerais. Revista Brasileira de Zootecnia, v.32, n.2, p.304-314, 2003. Disponível em: <http://www.scielo.br/ pdf/rbz/v32n2/16591.pdf>. Acesso em: 15 dez. 2010. doi: 10.1590/S1516-35982003000200008.

MATOS, R.S. et. al. Estudos dos efeitos genéticos e de meio ambiente sobre a produção de leite e gordura da raça Holandês no Estado do Rio Grande do Sul. Ciência Rural, v.27, n.3, p.465-471, 1997. Disponível em: <http://www.scielo.br/pdf/ cr/v27n3/a17v27n3.pdf >. Acesso em: $15 \mathrm{dez} .2010$. doi: 10.1590/S0103-84781997000300017.

MOLENTO, C.F.M. et al. Curvas de lactação de vacas holandesas do Estado do Paraná, Brasil. Ciência Rural, v.34, n.5, p.1585-1591, 2004. Disponível em: <http://www.scielo.br/ $\mathrm{pdf} / \mathrm{cr} / \mathrm{v} 34 \mathrm{n} 5 / \mathrm{a} 40 \mathrm{v} 34 \mathrm{n} 5 . \mathrm{pdf}>$. Acesso em: $15 \mathrm{dez}$. 2010. doi: 10.1590/S0103-84782004000500040.

PLOURD, R. Expect genetic changes. Dairy Herd Management, Saturday, July 01, 2000. Disponível em:<http:/ /www.dairyherd.com/directories.asp>. Acesso em: 8 de jan. 2008.

ROUGOOR, C.W. et al. Relationships between technical, economic and environmental results on dairy farms: an explanatory study. Livestock Production Science, v.47, n.1, p.235-244, 1997. Disponível em: <http://www.sciencedirect.com/ science?_ob=ArticleURL\&_udi=B 6T9B-3W0FR39F \&_ u s e r $=10 \&$ \& o v e r D a t e $=03 / 31 /$

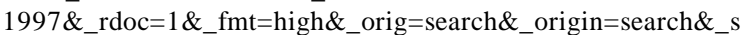
ort $=\mathrm{d} \& \_$docanchor $=\&$ view $=\mathrm{c} \& \_$acct $=\mathrm{C} 000050221 \& \_$version $=1 \&$ _ urlVersion=0\&_userid=10\&md5=a768e56ed $19 \mathrm{~d} 8 \mathrm{c} 78 \mathrm{cadf} 0372 \mathrm{fbf} 19$ f0b\&searchtype=a>. Acesso em: 15 dez. 2010. doi:10.1016/ S0301-6226(96)01408-X.

SAS. SAS INSTITUTE. Statistical analisis system. User's guide. Version 6.08. 4 ed. North Caroline, 1997. 846p.

WINDIG, J.J. et al. Genetic correlation between milk production and health and fertility depending on herd environment. Journal of Dairy Science, v.89, n.5, p.1765-1775, 2006. Disponível em: <http://www.journalofdairyscience.org/article/ PIIS0022030206722457/fulltext>. Acesso em: 15 dez. 2010. doi:10.3168/jds.S0022-0302(06)72245-7.

WOLFF, M.C. et al. Fatores ambientais sobem a idade ao primeiro parto, dias abertos e intervalo entre partos em vacas da raça holandesa na bacia leiteira de Castrolanda, Estado do Paraná. Archives of Veterinary Science, v.9, n.2, p.35-41, 2004.

YEREX, R.P. et al. Effects of selection for body size on feed efficiency and size of Holstein. Journal of Dairy Science, v.71, n.5, p.1355-1360, 1988. Disponível em: <http:// download.journals.elsevierhealth.com/pdfs/journals/0022-0302/ PIIS0022030288796939.pdf>. Acesso em: 15 dez. 2010. doi: 10.3168/jds.S0022-0302(88)79693-9.

ZWALD, N.R. et al. Genetic selection for helth traits using producer-recorded data. II. Genetic correlations, disease probabilities and relationship with existing traits. Journal of Dairy Science, v.87, n.12, p.4295-4302, 2004. Disponível em: <http:// www.journalofdairyscience.org/article/PIIS0022030204735742/ fulltext>. Acesso em: 15 dez. 2010. doi:10.3168/jds.S00220302(04)73574-2. 